\title{
Transalpina
}

TRANSALPINA Études italiennes

\section{1 | 2018}

Entre France et Italie : échanges et réseaux intellectuels au XIX $\mathrm{X}^{\mathrm{e}}$ siècle

\section{Michela Dota, «La vita militare » di Edmondo De Amicis. Storia linguistico-editoriale di un best seller postunitario}

Matteo Grassano

\section{OpenEdition}

\section{Journals}

\section{Edizione digitale}

URL: http://journals.openedition.org/transalpina/372

DOI: $10.4000 /$ transalpina.372

ISSN: 2534-5184

\section{Editore}

Presses universitaires de Caen

Edizione cartacea

Data di pubblicazione: 1 octobre 2018

Paginazione: $241-243$

ISBN: 978-2-84133-900-6

ISSN: 1278-334X

\section{Notizia bibliografica digitale}

Matteo Grassano, « Michela Dota, «La vita militare » di Edmondo De Amicis. Storia linguistico-editoriale di un best seller postunitario », Transalpina [Online], 21 | 2018, online dal 19 décembre 2019, consultato il 22 novembre 2020. URL : http://journals.openedition.org/transalpina/372 ; DOI : https://doi.org/ 10.4000/transalpina.372 
Michela Dota, «La vita militare» di Edmondo De Amicis. Storia linguistico-editoriale di un best seller postunitario, Milano, Franco Angeli, 2017, $351 \mathrm{p}$.

Il 19 agosto 1868 Edmondo De Amicis scriveva a Emilia Toscanelli Peruzzi: «È uscito finalmente il libro la Vita militare. Gliene ho spedito quattro copie. I librai di Firenze ne hanno fatto subito uno spaccio molto lusinghiero per me. Il generale Bariola mi ha scritto una lettera gentilissima che farà parte dell'archivio. Un'altra me ne scrisse il Direttore del Conte di Cavour, più gentile ancora, ma anche questa la riserbo per lei» (p. 264). Questi primi e positivi riscontri furono forieri di un successo che si concretizzò nei mesi successivi e che consacrò letterariamente il nome del giovane scrittore.

All'articolata vicenda dei bozzetti deamicisiani è dedicato il prezioso volume di Michela Dota, "La vita militare» di Edmondo De Amicis. Storia linguistico-editoriale di un best seller postunitario, che, coniugando la ricerca d'archivio con quella testuale, illumina l'evoluzione di un'opera iniziata sulle colonne dell' «Italia militare » e della «Gazzetta d'Italia» tra il 1867 e il 1868, e poi proseguita in volume fino alla terza edizione del 1880 .

Lo studio della storia editoriale e linguistica della Vita militare non può prescindere, come provano i primi due capitoli del libro, dalla « ricostruzione dell'ambiente socio-politico e culturale del salotto fiorentino frequentato dallo scrittore» (p. 9). In particolare, nel primo capitolo l'autrice evidenzia, sulla scorta del rapporto di De Amicis con Emilia Peruzzi, le linee guida della formazione ricevuta dallo scrittore: è in questo periodo che prende forma quel canone linguistico-letterario che, molti anni dopo, sarà esplicitato nell'Idioma gentile (1905). Per quanto riguarda le fonti utilizzate dalla Dota, occorre segnalare subito la lunga Appendice posta in fondo al libro che riproduce molte lettere di De Amicis alla signora Peruzzi, alternandole in modo efficace ad appunti tratti dalle agende private della donna pisana.

Il secondo capitolo conduce il lettore a scoprire le vicissitudini editoriali della Vita militare, ripercorrendo la nascita dei singoli bozzetti, la loro pubblicazione in rivista e la loro revisione in vista delle tre edizioni (1868, 1869 e 1880), di cui si sottolineano man mano le caratteristiche peculiari. Tramite la consultazione di varie fonti epistolari e l'interpretazione dei testi, Michela Dota ricostruisce l'influenza ideologica esercitata dal salotto Peruzzi sulle prime due edizioni dell'opera, le cui bozze passarono a più riprese sotto gli occhi e la penna della signora Emilia e di altri frequentatori dell'Antella, come Marco Tabarrini. Con la Vita militare, infatti, «De Amicis invera il progetto cullato dall'officina Peruzzi di produrre un buon libro di letteratura popolare educativa» (p. 243). Le correzioni alla terza edizione 
sono, invece, imputabili alla sola volontà autoriale: benché restino saldi gli ideali di base dell'opera, gli interventi correttori limano il moralismo di alcuni bozzetti e, più in generale, «depauperano il testo dai legami biografici e in parte culturali col salotto Peruzzi» (p. 10o).

L'importanza dei risultati conseguiti dall'autrice nel suo lavoro deve moltissimo alla magistrale analisi della lingua dei bozzetti militari, escussi nelle varie edizioni, che occupa i due capitoli successivi. All'esame della grafia, della fonetica e della morfologia segue, in una seconda parte, quello del lessico, della sintassi, della testualità e dello stile: entrambi sono condotti in una prospettiva diacronica che dà conto dell'evoluzione testuale e che dialoga continuamente tanto con altre opere deamicisiane quanto con strumenti e studi linguistico-lessicografici. L'argomentazione non corre il rischio descrittivo di una fenomenologia in cui i tratti rilevanti si perdono tra altri tipici della prosa del secondo Ottocento di ispirazione toscanista; al contrario, riesce a porre l'accento, di volta in volta, sugli aspetti distintivi delle scelte deamicisiane e a rimarcarne la funzione.

All'interno del complesso processo evolutivo dei testi sono senza dubbio individuabili alcuni punti fermi. Innanzitutto, è opportuno rilevare la tensione verso «l'etica della lingua» (p. 232), verso una scrittura animata da un afflato pedagogico e dalla volontà di coinvolgimento emotivo del pubblico medio. Come scrive l'autrice, "tutte e tre le edizioni conservano gli espedienti retorici che agevolano la processabilità del testo, nonché la sua memorabilità, come i chiasmi e i climax e tutte le figure di ripetizione e di ordine (anafore, polittoti e anadiplosi), applicate nelle cerniere testuali tra tema e rema" (ibid.). Al contempo, è fondamentale l'individuazione di un movimento correttorio teso al decrescimento della letterarietà - già riscontrabile nel passaggio dalle prime prove del 1867 alla prima edizione - che manifesta la necessità del giovane De Amicis di liberarsi dal patrimonio linguistico tradizionale appreso sui banchi di scuola. Tale esigenza conduce lo scrittore alla ricerca di una lingua media e di grande accessibilità che, a partire dalla seconda edizione, determina anche la caduta di molti fiorentinismi marcati non integrabili in un tessuto linguistico omogeneo.

Gli aspetti finora ricordati non possono che rimandare al confronto del giovane scrittore ligure-piemontese con la lingua del salotto Peruzzi e alla riflessione sulla teoria manzoniana dell'Uso, elaborata in forma definitiva nella Relazione del 1868. Da questo punto di vista l'analisi della Dota permette di definire, almeno per il periodo giovanile, i confini del manzonismo deamicisiano, mostrando come nell'autonomia di alcune scelte si rifletta la presa di coscienza, da parte di De Amicis, della propria individualità di scrittore: «Ecco dunque profilata la maggiore distanza tra De Amicis e 
Manzoni: De Amicis non rigetta l'allotropia, la considera una risorsa. Lo scarto tra la formazione illuministica del milanese e quella prettamente romantica del pedemontano è forse responsabile di questa differenza, che costituisce il nucleo linguistico non manzoniano, senza però contraddire la tensione etica della lingua, in Manzoni appoggiata anche dalla rinuncia ai doppioni» (p. 240).

In conclusione, il grande merito di Michela Dota, il cui libro diverrà sicuramente un testo indispensabile per gli studi linguistici deamicisiani, non è solo quello di aver ricostruito con accuratezza la vicenda editoriale della Vita militare nello stretto dialogo tra i membri del salotto Peruzzi, ma è anche quello di aver individuato, attraverso la ricchezza degli spogli, la centralità di quella prima prova letteraria nella messa a punto di una lingua piana e viva che, nei decenni successivi, avrebbe fatto la fortuna della prosa deamicisiana. Ė significativo notare che lo stesso De Amicis riconobbe l'esemplarità del proprio apprendistato linguistico, ponendolo silenziosamente alla base della sua ultima grande opera, quel «singolare manuale di glottodidattica che è L'idioma gentile» (p. 35): come dimostrano i puntuali riferimenti forniti dall'autrice, molte delle proposte linguistiche dell'Idioma recuperano, insieme all'apparato ideologico, il lavorio correttorio della Vita militare, a dimostrazione dell'assoluta rilevanza di quella prima esperienza di scrittura.

Matteo Grassano

\section{Patrizia Gabrielli, La guerra è l'unico pensiero che ci domina tutti. Bambine, bambini, adolescenti nella Grande guerra, Soveria Mannelli, Rubbettino, 2018, 193 p.}

Avec cet ouvrage, Patrizia Gabrielli apporte une contribution originale à l'immense dossier critique réuni par les historiens à l'occasion du centenaire de la Grande Guerre en se penchant sur la participation au conflit de la population «non adulte»: celles des enfants et des adolescents pris dans les engrenages d'une machine qui va profondément modifier leurs vies. Dans ce but, elle s'est appuyée sur une vaste documentation, en explorant comment la «culture de guerre» a investi d'une part la vie collective des jeunes générations par le biais de l'école, de la littérature pour la jeunesse, de la propagande, des rites et des cérémonies, et de l'autre leur vie privée, que nous révèlent les écritures du moi comme les lettres et les journaux intimes, conservés dans des archives telles que l'Archivio ligure della scrittura popolare, l'Archivio del Museo Storico di Trento et l'Archivio Diaristico nazionale de Pieve Santo Stefano, avec lequel l'auteure collabore. 